\title{
Vaccination Programs among Urban Homeless Populations: A Literature Review
}

\section{Stephen P. Wood*}

Nurse Practitioner, Winchester Hospital, Department of Emergency Medicine, Winchester, MA, USA

\begin{abstract}
Vaccination programs are an important component of public health initiatives and preventative medicine. This is particularly true in an urban environment where such factors as density, sanitation and pollution increase exposure to a variety of communicable disease. The most vulnerable populations such as slum-dwelling poor, homeless individuals and the elderly-poor are both more prone to exposure to communicable disease and to having a more limited access to the receipt of appropriate vaccinations to prevent them. A variety of programs have been utilized to maximize delivery of important vaccines to homeless individuals. Monetary incentives, education and ease of access are components of some of the more successful vaccination programs. This paper will provide a literature review of barriers to vaccination in homeless populations, programs and initiatives as well as future directions for vaccination programs.
\end{abstract}

Keywords: Homeless; Vaccinations; Vaccines; Hepatitis; Influenza

\section{Introduction}

Vaccination programs are an important component of public health initiatives and preventative medicine. This is particularly true in an urban environment where such factors as density, sanitation and pollution increase exposure to a variety of communicable diseases. The most vulnerable populations such as slum-dwelling poor, homeless individuals and the elderly-poor are both more prone to exposure to communicable disease and to having a more limited access to the receipt of appropriate vaccinations to prevent them $[1,2]$. In addition, certain characteristics such as mistrust of health care workers, mental illness and substance abuse may be barriers to participation in vaccination programs $[2,3]$. A variety of outreach programs have attempted to identify certain predictive characteristics that can improve the delivery of vaccinations to these populations. The purpose of this review is to investigate the literature regarding the urban homeless and vaccinations, focusing on access and delivery of vaccination programs in this population.

\section{Homelessness defined}

Homelessness is defined by the United Nations as those households without a shelter that would fall within the scope of living quarters. They carry their few possessions with them, sleeping in the streets, in doorways or on piers, or in another space, on a more or less random basis [4]. This was further defined by the United Nations Economic Commission for Europe Conference of European Statisticians into two countable groups; (1) primary homeless individuals who are persons living in the streets without a shelter that would fall within the scope of living quarters and (2) secondary homeless individuals who are persons with no place of usual residence who move frequently between various types of accommodations including dwellings, shelters and institutions for the homeless or other living quarters [5]. It is a worldwide problem with a conservative estimate of 3 billion homeless individuals across the globe, with many thousands if not millions more likely uncounted or unaccounted for. In the United States alone there are approximately 670,000 homeless individuals. Homeless populations often live on the street, in shelters or in squatters housing that characteristically are exposed to harsh weather elements, are crowded and have poor sanitation. Alcoholism and drug addiction is a problem for many homeless individuals and are risk factors for diseases such as hepatitis and human immunodeficiency virus (HIV) that can affect immunocomptence $[3,6]$. In addition a variety of other comorbidities are common in the homeless population such as obesity, malnutrition and diabetes mellitus. Access to clean water, healthy and fresh foods and basic hygiene are frequently lacking as well. Thus homeless individuals are a population that is particularly prone to a variety of communicable disease for multifactorial reasons.

\section{Vaccinations in homeless populations}

Vaccinations are of particular importance in vulnerable populations such as the homeless. This population is often exposed to harsh weather elements, crowding in shelters, and may have poor health secondary to medical comorbidities. Access to healthcare may be disparate and some individuals may not seek medical attention due to cost, concern for autonomy, distrust and/or mental illness. Issues such as unprotected sex, intravenous drug use (IVDU), and alcoholism also contribute to an increased risk in preventable diseases in this population. There is a growing body of literature on both the need and feasibility for vaccination programs among homeless individuals. That literature will be reviewed here to investigate the need and feasibility of vaccination programs in homeless individuals with a focus on the most widely studied vaccines, hepatitis B vaccine and influenza vaccine.

\section{Hepatitis B Vaccination}

Hepatitis B is a DNA virus of the Hepadnavirus family [7]. It is spread via blood and other body fluids to include semen and vaginal secretions. There are four known serotypes and eight known genotypes of the HBV which contributes both to varying clinical manifestations and drug resistance [8]. Pathologically the HBV virus infects

*Corresponding author: Stephen P. Wood, 69 Pleasant St. Boston, MA 02125 USA, Tel: 781-354-6422; E-mail: wood.s@husky.neu.edu

Received December 11, 2011; Accepted October 27, 2012; Published October 30, 2012

Citation: Wood SP (2012) Vaccination Programs among Urban Homeless Populations: A Literature Review. J Vaccines Vaccin 3:156. doi:10.4172/21577560.1000156

Copyright: @ 2012 Wood SP, et al. This is an open-access article distributed unde the terms of the Creative Commons Attribution License, which permits unrestricted use, distribution, and reproduction in any medium, provided the original author and source are credited. 
hepatocytes where it will replicate with resultant cell death via either cell lysis or apoptosis. The clinical manifestations are non-specific and can include the spectrum of asymptomatic disease, malaise, anorexia, fever, abdominal pain, myalgias, jaundice and rarely fulminant hepatitis [9]. The illness can be self-limiting, with almost $95 \%$ of patients recovering within 1 year of onset, although some individuals may develop relapses or the chronic form of disease $[7,10]$. Chronic $\mathrm{HBV}$ is of concern because it can lead to chronic illness, cirrhotic liver disease and malignancy [11]. Risk factors for HBV include IVDU, men who have sex with men (MSM), vertical transmission and heterosexual sex with a HBV carrier. There is a recombinant 3 dose vaccines with a $95 \%$ efficacy rate and 20 years or more duration of action that is FDA approved in the United States. The cost of the 3-vaccine series is estimated to be between $\$ 75$ to $\$ 165$ dollars for the series [12]. It is an important vaccine in the prevention of $\mathrm{HBV}$ with rates of decline in disease burden of $80 \%$ since initiation of vaccination program [13]. Most vaccination campaigns have been targeted towards infants and adolescents as well as healthcare workers. High risk groups, including many homeless individuals, are a vital component to reducing the incidence of HBV infection and transmission and several programs have been investigated to evaluate some of the barriers and obstacles to vaccination programs in this population.

Rates of Hepatitis B virus (HBV) have historically been high among homeless populations. According to the Center for Disease Control (CDC) the rate of HBV among homeless individuals ranges between 17 $31 \%$ [13]. Both Lum and Klinkenberg found that this rate approached $32 \%$ among individuals that were homeless with concurrent mental illness and substance abuse. Thus vaccine programs in this population are critical. At the same time, transiency, incarceration, drug use and mental illness can make it difficult to complete a 3 injection series within a 6 month period. As a result there have been several efforts to initiate and investigate the feasibility and barriers to HBV vaccination programs among homeless populations.

Nyamathi et al. [2] investigated the feasibility of a HBV vaccination program in homeless adults (age 18-65 years of age) from homeless shelters, detox treatment centers and the street in the "Skid Row" area of Los Angeles. They performed a randomized controlled trial of 3 different interventions to evaluate the feasibility of a HBV program in these individuals. The interventions included an intensive nurse case managed program (NCMP) that provided hepatitis and HIV education, seven sessions of education regarding self-esteem and coping skills, as well as monetary compensation (\$5) for each dose received as well as community outreach tracking. This was compared to a program of education, monetary incentives and tracking (SIT) as well as a third arm of just education and monetary incentives (SI). They enrolled 865 homeless adults and found that there was an almost 2 fold increase in completion of the 3 dose series with the nurse case management intervention. Overall rates of completion were 68\% (NCMP), $61 \%$ (SIT) and 54\% (SI) with the most likely to complete being older age, female sex and poorer health.

A follow-up study in 2010 by Stein and Nyamathi further investigated the NCMP group to determine if those most at risk, i.e. the subjects who were currently involved in unprotected sexual behaviors, needle sharing and prison history, were also those least likely to complete the 3 dose series. The enrollment included subjects from the NCMP arm of the prior study and evaluated risk-behaviors with a survey investigating needle sharing, sexual practices and prison history. Only a prior history of incarceration was associated with a modest risk for incompletion, demonstrating that increased risk for HBV was not correlated with program rejection [13]. They hypothesize that a history of incarceration is a marker of psychosocial impairment, poverty, mental illness and maladaptive behaviors, all of which can be associated with a low probability of completing a vaccination program. Greater coping skills and social support, an intervention that was provided and supported in the case management model, was positively correlated to increased completion. This is encouraging in that this is a feasible and important intervention that likely has an impact not only on vaccination rates but on other issues faced by homeless individuals as well.

Homeless urban youth are another group at substantial risk for $\mathrm{HBV}$ with high rates of substance abuse, risky sexual behaviors as well as tattooing and piercing in unsanitary environments. Haley et al. (1998) investigated completion of $\mathrm{HBV}$ vaccination in urban homeless youth (under the age of 25 years). This study involved a three-prong approach including an outreach and education program, the organization of widespread vaccination clinics and an active tracking and follow-up outreach program. Three hundred and eighty three participants met the inclusion criteria and of these $50.4 \%$ completed all three doses of vaccine. The positive correlates for receiving all three doses were female sex and involvement in prostitution. No differences were seen between those who used and those who did not use intravenous drugs, among men who have sex with men and men who did not have sex with men.

\section{Influenza}

Influenza is an upper respiratory illness caused by several viral strains of the orthomyxoviridae family (Cox, Subbarao, 1999). Transmission of influenza can occur via respiratory droplets and aerosols, as well as contact with nasal secretions, contaminated utensils or surfaces and in some cases with bird droppings. The clinical manifestation of influenza typically consists of fever, malaise, myalgias, non-productive cough and occasionally other symptoms to include sore throat, rhinorrhea and abdominal pain. Influenza is a worldwide concern, with typical seasonal flu strains claiming more than 250,000 - 500,000 lives annually. There have been well documented epidemics and pandemics that have been even more devastating. The most recent pandemic was the 2009 H1N1 strain, which, while most cases where self-limiting and mortality was low, was quite virulent and spread rapidly around the globe. Non-pharmacological management of the spread of influenza includes covering coughs and sneezes as well as excellent hand sanitation and isolation and/or masks for those that are ill with confirmed or suspected illness. Annual vaccination is an important pharmacological intervention for the prevention of disease. A trivalent, inactivated vaccination is available and is administered on an annual basis with viral constituents generally including most recent infectious or circulating strains. It is administered several months prior to influenza season with an efficacy of approximately $60-70 \%$ [14] The crowded streets and shelters and unsanitary environment that the homeless are often exposed to make non-pharmacological methods difficult. In addition, lack of access to primary care, as well as the issues related to mental illness, substance abuse and distrust of healthcare providers may decrease vaccination rates among homeless populations.

Historically low vaccination rates combined with increased risks for illness and morbidity and mortality from influenza makes vaccination an imperative. A variety of measures that may work as general measures have included vaccination programs at religious centers, pharmacies, as standing orders in hospitals, visiting nurse programs or non-traditional vaccination programs at needle-exchange sites may have limitations when considering hard-to-reach and homeless populations. Buchner et al. in 2006 did a retrospective review of medical records of homeless 
individuals who visited a medical clinic and found that only $25 \%$ of these patients had received influenza vaccine. This was coupled with data that influenza or an influenza- like illness was the suspected cause of death in approximately $3.4 \%$ of deaths among homeless individuals in New York city [1].

In 2009 during the H1N1 pandemic, French investigators evaluated the effectiveness of a shelter-based vaccination program among homeless individuals. They provided the opportunity to refuse vaccination, accept vaccination or have an educational program prior to refusing or accepting vaccination. Their data revealed that $95 \%$ of the study subjects were aware of the potential pandemic, but that only $14 \%$ of the individuals in the shelter had received the seasonal influenza vaccine the year of the vaccine intervention. Only 1 of the 109 participants in the program had received the H1N1 vaccine and only $46 \%$ agreed to vaccination during the program [15]. While this number seems low given the knowledge base and free access, it was comparatively much higher than the vaccination acceptance rates of the non-homeless, general public of $10 \%$ during the same period. They postulate that the access to education, access to the vaccine and perceived increased risk contributed to these higher rates.

\section{Discussion}

Vaccinations are an important part of public health measures for infectious disease yet they are often not available to some of the most vulnerable populations. Homeless individuals have many factors that prevent appropriate vaccination to include substance abuse, mental illness and distrust of medical professionals. At the same time many homeless individuals are at greater risk for illness secondary to highrisk behaviours, substance abuse, malnutrition, co-morbidities and lack of access. A variety of methods have been attempted and evaluated for effectiveness. Simply offering a vaccine without other educational initiatives or incentives is the least likely to be successful. Educational incentives that allow individuals to discuss the concept of vaccines as well as the risks and benefits do incur some additional value to vaccination acceptance. Other incentives including monetary incentives have also been utilized with some success. Combining ease of access, education, self-esteem and coping strategies, and incentives is likely to be the best approach to vaccination administration programs in this population. It is clear that incarceration is also a risk factor for inadequate vaccination and this may be an area of further investigation for initiating vaccination programs as incarceration can be associated with or lead to homelessness. In addition, there needs to be ongoing research for other vaccines that could be of benefit to include pertussis, tetanus, meningococcemia and varicella. To date there are no studies or evaluations of these vaccines in homeless individuals.

Future direction for research in this area can target several areas of study. One example is investigating optimal locations for serving hard-to-reach individuals for vaccination programs including street vaccination, shelter vaccination and possibly novel locales such as bottle collection centres, liquor stores or public libraries. In addition, there is a need to determine what factors contribute to improved vaccination uptake, while maintaining a cost-effective delivery program. The papers investigated in this review evaluate self-esteem, incentives and educational programs, some of which can be labor and cost-intensive. It is imperative that further investigations evaluate the most effective means of carrying out vaccination programs with insight into cost and cost-effectiveness. Efficacy rates of vaccines are another important area of study. We have data on general efficacy rates but rates specific to homeless populations is not available. Whether or not these rates would differ are unknown but could be important when considering dosing schedules, delivery systems and vaccine preparations. It also would play a role in understanding the cost-effectiveness of vaccine delivery programs compared to the healthcare spending burden. Lastly, there is a need to investigate other vaccine types for diseases that are common in homeless populations, including pneumococcal, meningococcal, tetanus and pertussis vaccine programs. Important among these will be an introduction of an HIV vaccine and HIV vaccine studies, in a group that is vulnerable to this disease but may have barriers to acceptance including mistrust, substance abuse and mental illness. The homeless population is a high-risk group for communicable disease and it is an imperative that future research and implementation programs focuses on well-designed, validated methods for delivering vaccines to this at risk population.

\section{Results}

1. Bucher S, Brickner P, Vincent R (2006) Influenza like illness among homeless persons. Emerg Infect Dis 12: 1162-1163.

2. Nyamathi A, Liu Y, Marfisee M, Shoptaw S, Greqerson P, et al. (2009) Effects of a nurse-managed program on hepatitis $A$ and $B$ vaccine completion among homeless adults. Nurs Res 58: 13-22.

3. Larimer ME, Malone DK, Garder MD, Atkins DC, Burlingham B, et al. (2009) Health care and public service use and costs before and after provision of housing for chronically homeless persons with severe alcohol problems. JAMA 301: 1349-1357.

4. Department of Economic and Social Affairs, Statistics Division, Demographic and Social Statistics Branch (2004) United Nations Demographic Yearbook review: National reporting of household characteristics, living arrangements and homeless households: Implications for international recommendations. United Nations.

5. United Nations, Economic, Social Council (2009) Enumeration of Homeless People. Economic Commission for Europe Conference of European Statisticians, Geneva.

6. Wenzel SL, Green HD Jr, Tucker JS, Golinelli D, Kennedy DP, et al. (2009) The social context of homeless women's alcohol and drug use. Drug Alcohol Depend 105: 16-23.

7. Garfein R, Bower W, Loney C, Hutin YJ, Xia GL, et al. (2004) Factors associated with fulminant liver failure during an outbreak among injection drug users with acute hepatitis B. Hepatology 40: 865-873.

8. Sato S, Suzuki K, Akahane Y, Akamatsu K, Akiyama K, et al. (1995) Hepatitis $B$ virus strains with mutations in the core promoter in patients with fulminan hepatitis. Ann Intern Med 122: 241-248.

9. Liaw Y, Tsai S, Sheen I, Chao M, Yeh CT, et al. (1998) Clinical and virologica course of chronic hepatitis $B$ virus infection with hepatitis $C$ and $D$ virus markers. Am J Gastroenterol 93: 354-359.

10. Yotsuyanagi H, Yasuda K, lino S, Moriya K, Shintani Y, et al. (1998) Persisten viremia after recovery from self-limited acute hepatitis B. Hepatology 27: 13771382.

11. Kumar M, Satapathy S, Monga R, Das K, Hissar S, et al. (2007) A randomized controlled trial of lamivudine to treat acute hepatitis B. Hepatology 45: 97-101.

12. Hepatitis B Vaccine (2009) Retrieved from: http://www.hepb.org/hepb/vaccine information.htm.

13. Stein J, Nyamathi A (2010) Completion and subject loss within an intensive hepatitis vaccination intervention among homeless adults: the role of risk factors, demographics and psychosocial variables. Health Psychology 29: 317 323

14. US Centers for Disease Control and Prevention. Interim guidance on the use of influenza antiviral agents during the 2010-2011 influenza season.

15. Nougairede A, Lagier JC, Ninove L, Sartor C, Badiaga S, et al. (2010) Likely correlation between sources of information and acceptability of $\mathrm{A} / \mathrm{H} 1 \mathrm{~N} 1$ swineorigin influenza virus in Marseille, France. PLoS One 5: e11292. 